\title{
Middle cerebral artery thrombus susceptibility-weighted imaging mapping predicts prognosis
}

\author{
Mei-Zhu Zheng ${ }^{1 \#}$, Qing-Yuan Yang ${ }^{2 \#}$, Xiu-Di Lu ${ }^{3,4 \#}$, Si-Le Hu ${ }^{5}$, Chao Chai ${ }^{6}$, Wen Shen ${ }^{6}$, Bin-Ge Chang ${ }^{7}$, \\ Zhi-Yun Wang ${ }^{8}$, Shuang Xia ${ }^{6}$ \\ ${ }^{1}$ Radiological Department, Third Central Hospital of Tianjin, Tianjin Key Laboratory of Artificial Cell, Artificial Cell Engineering Technology \\ Research Center of Public Health Ministry, Tianjin 300170, China; ${ }^{2}$ Radiological Department, Tianjin Haihe Hospital, Tianjin 300222, China; \\ ${ }^{3}$ Radiological Department, First Central Clinical College, Tianjin Medical University, Tianjin 300381, China; ${ }^{4}$ Radiological Department, First \\ Teaching Hospital of Tianjin University of Traditional Chinese Medicine, Tianjin 300192, China; ${ }^{5}$ Intervention Division Department, Inner \\ Mongolia Autonomous Region People's Hospital, Hohhot 010020, China; ${ }^{6}$ Radiological Department, ${ }^{7}$ Neurosurgery Department, ${ }^{8}$ Neurological \\ Department, Tianjin First Central Hospital, Tianjin 300192, China
}

"These authors contributed equally to this work.

Correspondence to: Shuang Xia. Tianjin First Central Hospital, Tianjin 300192, China. Email: xiashuang77@163.com; Binge Chang. Tianjin First Central Hospital, Tianjin 300192, China. Email: changcheng1968@sina.com; Zhiyun Wang. Tianjin First Central Hospital, Tianjin 300192, China. Email:13820585625@163.com.

Background: Susceptibility weighted imaging and mapping (SWIM) of magnetic resonance imaging (MRI) is used to evaluate cerebral arterial thrombosis. The aim of this research was to assess susceptibility, length, and clot burden score (CBS) of thrombus in the middle cerebral artery (MCA) and their relationship with cerebral infarction and early clinical prognosis in patients with acute or subacute cerebral infarction.

Methods: In total, 56 patients with acute or subacute cerebral infarction (with the time from onset to admission less than $72 \mathrm{~h}$ ) and only unilateral MCA occlusion were included in the current study. All the patients had the corresponding susceptibility vessel sign (SVS) on susceptibility-weighted imaging (SWI). Parameters including susceptibility, length, and CBS of thrombus were obtained from SWI and SWIM. The differences in susceptibility of different portions of the thrombus were compared with each other by oneway ANOVA test. The relationship between susceptibility and stroke onset time was further analyzed by Spearman correlation analysis, in addition to the relationships between susceptibility, length, CBS, diffusionweighted imaging-Alberta stroke program early CT score (DWI-ASPECTS), and admission and discharge National Institutes of Health Stroke Scale (NIHSS).

Results: The susceptibility among different portions and different segments of thrombus showed no statistical difference. The susceptibility and length were weakly yet negatively correlated with DWIASPECTS $\left(\mathrm{r}_{\mathrm{s}}=-0.382,-0.457 ; \mathrm{P}=0.004,0.000\right)$. The susceptibility was weakly yet positively correlated with admission NIHSS and discharged NIHSS $\left(\mathrm{r}_{\mathrm{s}}=0.403,0.430 ; \mathrm{P}=0.002,0.001\right)$. CBS was weakly yet positively correlated with DWI-ASPECTS $\left(\mathrm{r}_{\mathrm{s}}=0.349 ; \mathrm{P}=0.008\right)$ and weakly yet negatively correlated with admission and discharged NIHSS $\left(\mathrm{r}_{\mathrm{s}}=-0.375,-0.335 ; \mathrm{P}=0.004,0.012\right)$.

Conclusions: The susceptibility remained consistent regardless of location, length, and onset time, which indicates that the thrombus composition was similar when detected on SWI less than $72 \mathrm{~h}$ from the onset. Susceptibility and CBS may help to predict clinical severity and short-term clinical prognosis to some extent.

Keywords: Stroke; thrombus; susceptibility; susceptibility-weighted imaging mapping; middle cerebral artery occlusion

Submitted Aug 12, 2019. Accepted for publication Aug 19, 2019.

doi: 10.21037/qims.2019.08.17

View this article at: http://dx.doi.org/10.21037/qims.2019.08.17 


\section{Introduction}

Thrombus formation of the intracranial vessels is the most common cause of acute cerebral infarction. Eighty percent of cerebral blood flow comes from the middle cerebral artery (MCA), which is the most preferred site of thrombosis leading to embolic brain infarction. Recanalization of the occluded blood vessels within $6 \mathrm{~h}$ by early thrombolytic or thrombectomy therapy can increase brain tissue reperfusion and save the ischemic penumbra, which is important to clinical prognosis (1). Some papers demonstrate that the composition of the thrombus can affect recanalization; for instance, red thrombus can be easily recanalized after thrombolytic or thrombectomy (2-4). In addition, the location, length of thrombus could influence the formulation of the treatment plan $(5,6)$. Therefore, timely thrombus detection and accurate assessment including location, length, and composition of thrombus play a crucial role in the choice of the treatment plan and in the clinical prognosis assessment.

Despite the fact that the thrombus of the intracranial vessels can be found in all three methods of digital subtraction angiography (DSA), magnetic resonance angiography (MRA), and computed tomography angiography (CTA), the questions about its composition and formation times still could not be answered (7-11). To solve these two problems, the application of susceptibilityweighted imaging (SWI) in the detection of thrombus in acute ischemic stroke is becoming an area of intense research interest (12-14). SWI can show an intravascular thrombus as a hypointense susceptibility vessel sign (SVS) which has a classical manifestation as a thickened and dark intravascular susceptibility artifact. SVS indicates the increment of locally increased deoxyhemoglobin from the red blood cells (RBCs) trapped in occlusive vessels $(4,13,15)$. SVS has been investigated in a large amount of research which supports the presence of acute or subacute thrombus $(16,17)$. Some studies have shown that SVS can provide useful information regarding the thrombus location, the presence of single or multiple thrombi (even in distal intracranial arteries), and the thrombus burden $(18,19)$. Linked to the local presence of intra-arterial thrombus, SVS is associated with arterial occlusion (20) and disappears with the occurrence of arterial recanalization $(14,21)$.

Susceptibility weighted imaging mapping (SWIM) is calculated based on the $3 \mathrm{D}$ multi-echo gradientecho sequence and combines both phase and magnitude information to quantify susceptibility distribution. It has been widely used in the evaluation of brain iron deposition in a variety of metabolic diseases (22-25), and the detection of microbleeds $(26,27)$ or oxygen saturation changing in stroke patients (28-30). However, there is no report focusing on the changes in thrombus susceptibility in MCA.

Based on the knowledge that the composition of thrombus is different and only the acute or subacute stage of the thrombus can show SVS on SWI, we hypothesized that the susceptibility not only could reflect the composition of thrombus but also predict the formation time of thrombus. Susceptibility, thrombus length, and clot burden score (CBS) might have correlations with clinical status and early prognosis.

In this paper, we tried to answer the following two questions: whether the susceptibility of thrombus in MCA varies between segments, portions, or stroke onset times; whether there are any relationships between susceptibility, thrombus length, CBS, diffusion-weighted imaging-Alberta stroke program early CT score (DWI-ASPECTS), and National Institutes of Health stroke scale (NIHSS).

\section{Methods}

\section{Patients}

The current study was approved by the local Institutional Review Board (IRB) of Tianjin First Central Hospital. Written consent signed by the patients or their relatives were stored in the hospital database to be reviewed. Clinical and imaging data of 153 patients with acute or subacute stroke (onset time was less than $72 \mathrm{~h}$ which was defined as the time interval from the onset of stroke symptoms to MRI examination) from November 2013 to December 2016 were collected and retrospectively analyzed. All the patients were treated with anticoagulation alone, without rTPA or thrombectomy. Inclusion criteria included the following: adult patients (over 18 years old); the time interval (from stroke onset to admission) less than $72 \mathrm{~h}$; complete MRI data including T1-weighted images (T1WI), T2-weighted images (T2WI), T2 fluidattenuated inversion recovery (T2 FLAIR), diffusionweighted images (DWI), time of flight-MRA (TOF-MRA), SWI, and complete clinical data including admission and discharged NIHSS; DWI restriction showing high intensity and corresponding ADC being low intensity; unilateral MCA occlusion in the TOF-MRA and SVS on SWI. Exclusion criteria included the following: patients with bilateral MCA occlusion or intracranial lesions, including 


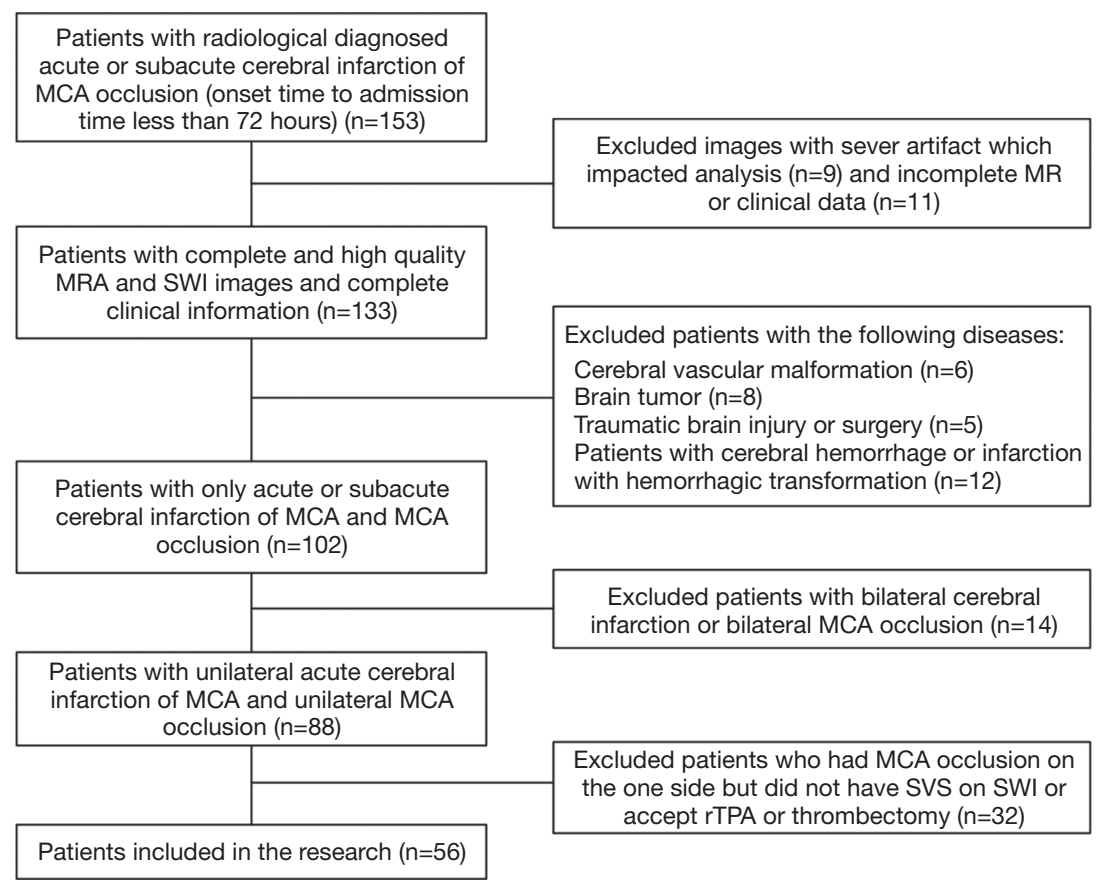

Figure 1 Flow chart for the patients inclusion and exclusion. MCA, middle cerebral artery; SWI, susceptibility-weighted imaging; SVS, susceptibility vessel sign; rTPA, recombinant tissue plasminogen activator.

cerebral vascular malformation, brain tumors, traumatic brain injury or surgery, cerebral hemorrhage, or infarction with hemorrhagic transformation. Detailed inclusion and exclusion criteria are shown in Figure 1. Finally, a total of 56 patients were included in this study.

\section{Image acquisition}

A MAGNETOM Trio Tim 3T MR scanner (Siemens Healthcare, Erlangen, Germany) with an 8-channel head coil was used for MRI examination. The examining sequences for all cases included T1WI, T2WI, T2 FLAIR, DWI, TOF-MRA, and SWI. The detailed sequences' information is summarized in Table 1.

\section{Imaging processing and analyzing}

Primary SWI data with complete phase and magnitude images were processed using one button with SMARTv1.0 (susceptibility mapping and phase artifacts removal tool; Detroit, MI, USA) software within 30 s. The processing steps are detailed below. First, the unwanted low signal regions outside the brain were removed by a Brain Extraction Tool (BET) in FMRIB Software Library
(FSL) (31). Second, the background phase was reduced by a $96 \times 96$ homo-dyne high-pass filter. Finally, SWIM data were generated by a truncated $\mathrm{k}$-space division with a regularization threshold of 0.1 (32). The SWIM data were then observed and measured by signal processing in nuclear magnetic resonance (SPIN) (Detroit, MI, USA) software. The portions of M1 (initial and horizontal segment), M2 (Sylvian segment), and M3 (cortical segment) were marked, and susceptibility was manually measured. The detailed measurements are displayed in Figure 2A. The susceptibility of each segment was used to calculate the overall mean susceptibility of the multi-segmental thrombus. Thrombus length and CBS were measured on the SWI MinIP images along the segment of thrombus $(5,33,34)$. The detailed measurements are shown in Figure 2B,C and Figure 3. DWI-ASPECTS was evaluated depending on the DWI and ADC images, according to the criteria of CT-ASPECTS $(35,36)$. All of the measurements were repeated 3 times to calculate an average value by a neuroradiologist of 10-year experience and one of 5-year experience who both specialize in diseases of the central nervous system. The two readers were blinded to clinical data. The mean value of the two readers would be used if there were good consistency between them. 
Table 1 Imaging parameters of MR sequences

\begin{tabular}{lcccccccc}
\hline MR sequences & TR $(\mathrm{ms})$ & TE $(\mathrm{ms})$ & Flip angle $\left(^{\circ}\right)$ & FOV $\left(\mathrm{mm}^{2}\right)$ & Resolution $\left(\mathrm{mm}^{3}\right)$ & Bandwidth $(\mathrm{Hz})$ & Slice & Acquisition time \\
\hline T1WI & 250 & 2.46 & 70 & $240 \times 240$ & $0.9 \times 0.8 \times 5$ & 330 & 20 & $1 \mathrm{~min} 6 \mathrm{~s}$ \\
T2WI & 6,000 & 93 & 120 & $220 \times 220$ & $0.7 \times 0.7 \times 5$ & 220 & 20 & $1 \mathrm{~min} 45 \mathrm{~s}$ \\
DWI & 4,000 & 96 & 90 & $230 \times 230$ & $0.6 \times 0.6 \times 5$ & 1,343 & 20 & $1 \mathrm{~min} 1 \mathrm{~s}$ \\
SWI & 27 & 20 & 15 & $230 \times 200$ & $0.5 \times 0.5 \times 2$ & 120 & 56 & $2 \mathrm{~min} 58 \mathrm{~s}$ \\
MRA & 22 & 3.59 & 18 & $240 \times 200$ & $0.8 \times 0.8 \times 0.8$ & 164 & 240 & $7 \mathrm{~min} 2 \mathrm{~s}$ \\
\hline
\end{tabular}

MR, magnetic resonance; TR, time of repetition; TE, time of echo; FOV, field of vision; T1WI, T1-weighted images; T2WI, T2-weighted images; DWI, diffusion-weighted images; SWI, susceptibility-weighted imaging; MRA, magnetic resonance angiography.
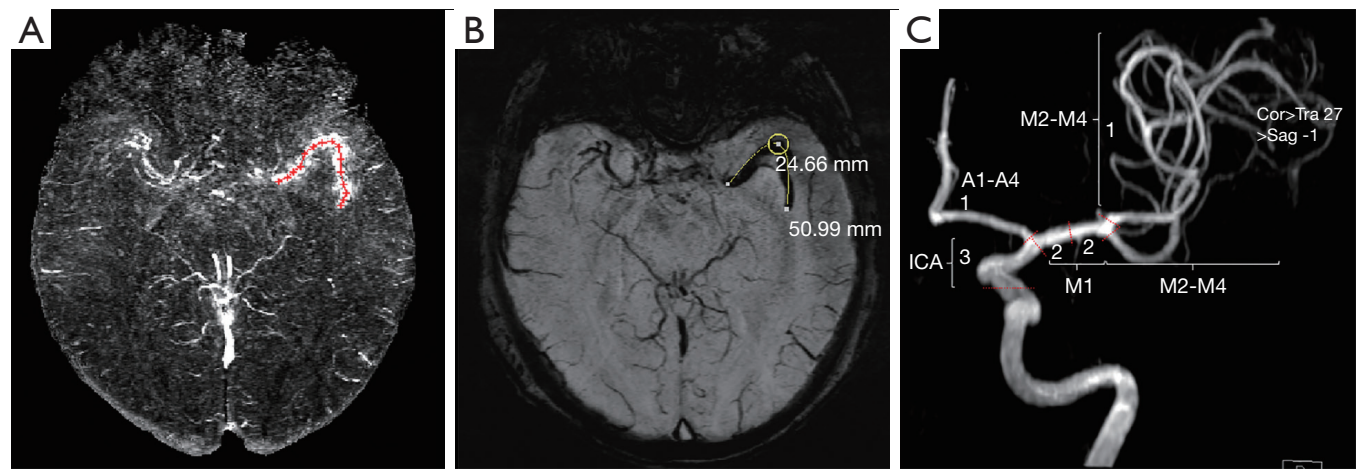

Figure 2 The detailed measurements. (A) The region of interest (ROI) was drawn in the center along the thrombus, the susceptibility value of each point could be calculated directly by the SPIN software to obtain the average value and standard deviation of the susceptibility values in the ROI finally (Red+ represents ROI). (B) The length of thrombus was measured 3 times on the susceptibility-weighted imaging (SWI) MinIP images and the mean value of these was recorded. The corresponding score was deducted from the thrombus at the corresponding position. (C) ROI along the susceptibility vessel sign (SVS) on SWIM was drawn to calculate the repetition of susceptibility 3 times, and the mean value was recorded. Clot burden score (CBS) was graded by the following standard [5]: a score of 10 is normal; 3 points (as indicated) are subtracted for SVS found in the supraclinoid internal carotid artery (ICA); 2 points for SVS in each of the proximal and distal halves of the middle cerebral artery (MCA) trunk (M1); 1 point for SVS in the A1-A4 segment; and 2 points for SVS in the M2-M4 branches.

\section{Statistical analysis}

The data of this study were analyzed by SPSS 22.0 software. The intraclass correlation coefficient (ICC) was performed to test the agreement of quantitative parameters between the two readers. If the ICC value was less than 0.4 , the consistency of reliability analysis was considered poor. If the ICC value was greater than 0.75 , the consistency was considered good (Table S1). The clinical variables and parameters were tested for normal distribution using the Kolmogorov-Smirnov test method (Table S2). If the values of susceptibility fit the normal distribution, mean \pm standard deviation (SD) was used to describe them, and one-way ANOVA test was used to separately compare the differences among the different locations and different segments. If not, median (P25, P75) and Kruskal-Wallis H tests were used. The Spearman correlation analysis was used to analyze the relationship between susceptibility and stroke onset time. The relationships between the length of thrombus, CBS, susceptibility, DWI-ASPECTS, admission NIHSS, and discharge NIHSS were analyzed by Spearman correlation analysis (Table S3). Before this step, the residual analysis was used to find whether there were any outliers. $\mathrm{P}<0.05$ was considered statistically significant. The correlation coefficient $\left(r_{s}\right)$ ranged from -1 to 1 . If $\left|r_{s}\right|$ was less than 0.3 , a poor correlation was concluded, if $\left|r_{s}\right|$ was larger than 0.3 but less than 0.5 , week relationship was concluded. If $\left|r_{s}\right|$ was over 0.5 

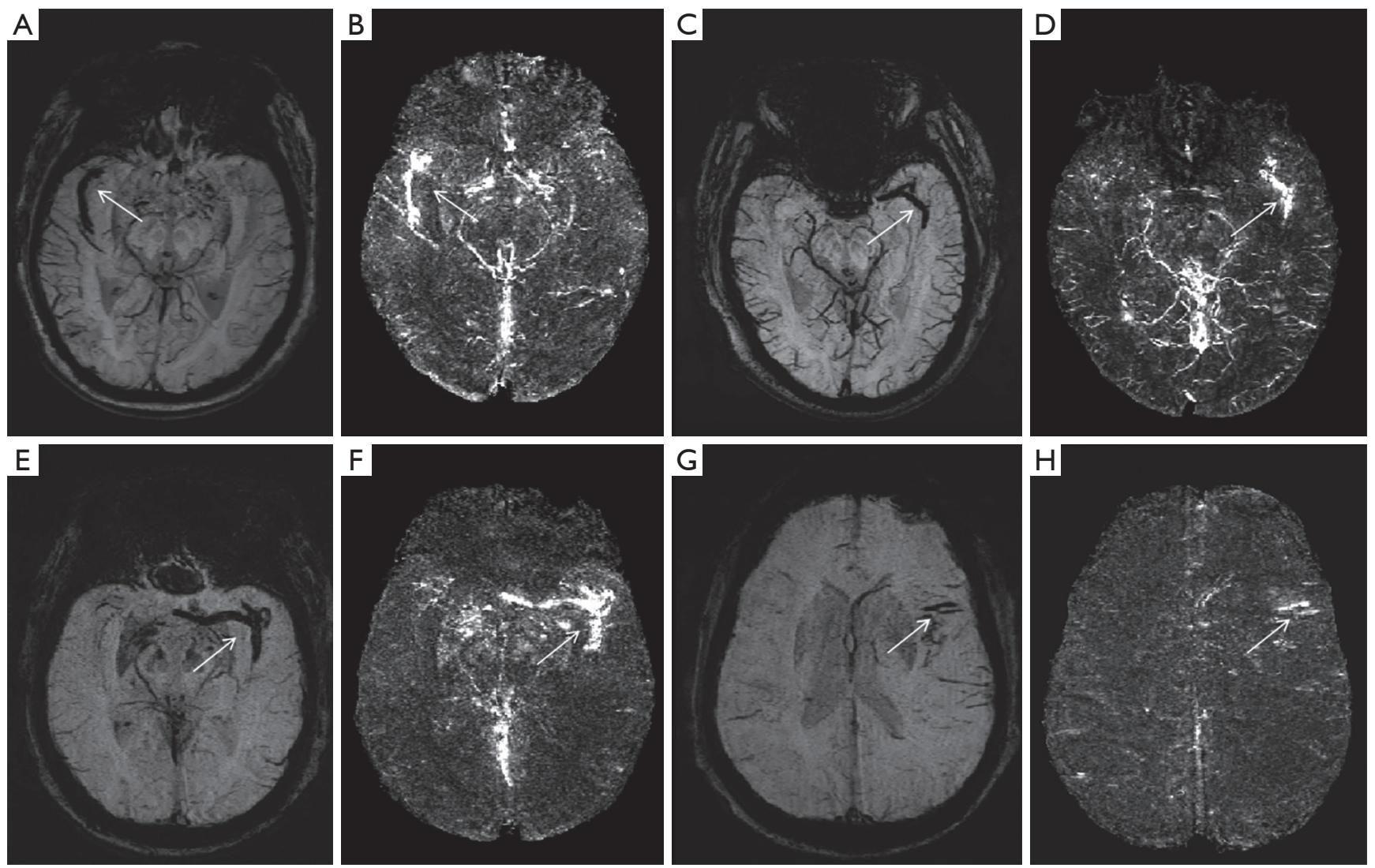

Figure 3 The SWI and SWIM images of thrombi at different sites. (A,B) Female, 66 years old. susceptibility-weighted imaging (SWI) MinIP images shows low signal (white arrow in A) and susceptibility weighted imaging and mapping (SWIM) shows high signal (white arrow in B) thrombus in the right middle cerebral artery (MCA) (M2) with clot burden score (CBS) recorded as "9"; (C,D) Male, 57 years old. SWI MinIP shows low signal (white arrow in C) and SWIM shows high signal (white arrow in D) thrombus in the left MCA branch segment (M1, M2) with CBS recorded as "8"; (E,F,G,H) Male, 84 years old. SWI MinIP shows multiple low signal (white arrow in E,G) and SWIM shows high signal (white arrow in F,H) thrombi in the left MCA branches (M1, M2, M3), with CBS recorded as "4".

but less than 0.8 , a moderate correlation was concluded. If $\left|\mathrm{r}_{\mathrm{s}}\right|$ was over 0.8 , a strong correlation was concluded.

\section{Results}

The baseline characteristics of the enrolled patients are summarized in Table 2. For the 56 patients, the median length of MCA thrombus was $16.13(10.58,29.03) \mathrm{mm}$. The mean susceptibility of thrombus was $387.168 \pm 104.612 \mathrm{ppb}$. The median CBS related to the portion of thrombus was 8 $(6,8)$. The median onset time was $21(14,28.3) \mathrm{h}$.

\section{The comparison of susceptibility among thrombi of different segments}

Based on the locations of SVS on SWI, MCA thrombi were segmented. There were 31 (55.36\%) cases with 1 segment, 19 cases $(33.93 \%)$ with 2 segments, and 6 cases $(10.71 \%)$ with 3 segments. Among all thrombi, 17 segments were located at M1, 42 were located segments at M2, and 28 segments were located at M3. The susceptibility among different portions and different segments of thrombi showed no statistical difference (All $\mathrm{P}>0.05$ ) (Table 3).

\section{The relationship between susceptibility and onset time, length of thrombus, and CBS}

When there were 2 or 3 thrombi in different segments of MCA in 1 patient, the mean value of thrombi susceptibilities was considered as the mean susceptibility of this patient because the consistent susceptibility of each segment was proven. The mean susceptibility had no correlations with 
Table 2 Baseline characteristic of the enrolled patients ( $\mathrm{n}=56)$

\begin{tabular}{|c|c|}
\hline Variables & Value \\
\hline Age (years) ${ }^{\mathrm{a}}$ & $64.5 \pm 13.9$ \\
\hline \multicolumn{2}{|l|}{ Gender, n (\%) } \\
\hline Male & $36(64.28)$ \\
\hline Female & 20 (35.72) \\
\hline \multicolumn{2}{|l|}{ Risk factors, n (\%) } \\
\hline Hypertension & $37(66.07)$ \\
\hline Diabetes & $12(21.43)$ \\
\hline Coronary heart disease & $20(35.71)$ \\
\hline Onset time $\mathrm{b}^{\mathrm{b}}$ (hours) & $21(14,28.3)$ \\
\hline \multicolumn{2}{|l|}{$\mathrm{NIHSS}{ }^{b}$} \\
\hline Admission & $10.5(4,19)$ \\
\hline Discharge & $12(5.75,15.25)$ \\
\hline DWI-ASPECTS ${ }^{b}$ & $6(4,7)$ \\
\hline \multicolumn{2}{|l|}{ Thrombus side, $\mathrm{n}(\%)$} \\
\hline Left & $27(48.21)$ \\
\hline Right & $29(51.79)$ \\
\hline
\end{tabular}

a, continuous numerical variables fit normal distribution; b, continuous numerical variables did not fit normal distribution. NIHSS, National Institutes of Health Stroke Scale; DWIASPECTS, diffusion-weighted imaging-Alberta stroke program early CT score.

onset time, thrombus length, and CBS $(\mathrm{P}=0.803,0.110$, 0.611) (Figure S1).

\section{The effects of susceptibility, length of thrombus, and CBS on clinical severity and early clinical prognosis}

The susceptibility was weakly yet negatively correlated with DWI-ASPECTS $\left(\mathrm{r}_{\mathrm{s}}=-0.382, \mathrm{P}=0.004\right)$ (Figure $4 A$ ), and weakly positively correlated with NIHSS at admission and discharge $\left(\mathrm{r}_{\mathrm{s}}=0.403, \mathrm{P}=0.002 ; \mathrm{r}_{\mathrm{s}}=0.430, \mathrm{P}=0.001\right)$ (Figure 4B,C).

A negative correlation was found between the length of MCA thrombus and DWI-ASPECTS $\left(\mathrm{r}_{\mathrm{s}}=-0.457, \mathrm{P}=0.000\right)$ (Figure 5) (Figure S2). There was no statistically significant correlation between the length of MCA thrombus and admission and discharge NIHSS $\left(\mathrm{r}_{\mathrm{s}}=0.295,0.224 ; \mathrm{P}=0.026\right.$, 0.100) (Figure S2).

There was a weak positive correlation between CBS and DWI-ASPECTS $\left(\mathrm{r}_{\mathrm{s}}=0.349, \mathrm{P}=0.008\right)$, but a weak negative correlation with the admission and discharge NIHSS $\left(\mathrm{r}_{\mathrm{s}}=-0.375,-0.335 ; \mathrm{P}=0.004,0.012\right)$ (Figure 5) (Figure $S 3)$. The CBS was weakly yet negatively correlated with thrombus length of MCA $\left(\mathrm{r}_{\mathrm{s}}=-0.382, \mathrm{P}=0.004\right)$ (Figure 5) (Figure S2).

\section{Discussion}

There were two main findings in this study. Firstly, all the susceptibility remained consistent regardless of different segments, portions, or onset time. Secondly, susceptibility and CBS were related to cerebral infarction severity, and they could, to some extent, predict clinical severity and short-term clinical prognosis.

In this study, the susceptibility among different segments and different portions of MCA thrombi showed no statistical difference but did indicate the similar component of the whole thrombus. Aoki et al. (36) suggested that the presence of SVS may represent acute or subacute thrombi, given the time required from oxyhemoglobin to deoxyhemoglobin within hyperacute clots. Susceptibility was related to the component of paramagnetic deoxyhemoglobin, which can be seen in the acute or subacute stage of thrombus formation. Therefore, the results support the notion that thrombus is formed in a similar processing stage of platelet aggregation and blood cell coagulation.

We found no relationship between the susceptibility and the onset time. This might indicate that there was no difference for the susceptibility of thrombus when detected on SWI regardless of being acute or subacute stroke (less than $72 \mathrm{~h}$ ). In other words, it implies that the amount of deoxyhemoglobin in the thrombus remained similar. However, the formation and evolution of thrombus are extremely complex $(37,38)$, and the onset time we acquired cannot fully represent the real-time of thrombus formation. The relationship between susceptibility and thrombosis evolution remains to be further studied. Susceptibility was weakly correlated with DWI-ASPECTS, admission, and discharge NIHSS in the current study. This result might indicate that susceptibility does not only reflect the component of thrombus but also may have a relationship with the degree of clinical severity.

MCA thrombus load mainly depended on thrombus length, location, and composition. We found that the length of the MCA thrombus was weakly yet negatively correlated with DWI-ASPECTS. Studies (36) have found that DWIASPECTS has a close correlation with the injury volume and is also associated with early prognosis after intravenous 
Table 3 Susceptibility $(\mathrm{ppb})($ mean \pm SD) of the middle cerebral artery (MCA) thrombi in different groups

\begin{tabular}{lcccccc}
\hline $\begin{array}{l}\text { Segments/sites } \\
\text { and statistics }\end{array}$ & Single segment $(n=31)$ & Two segments $(n=38)$ & Three segments $(n=18)$ & Total & $P$ & \\
\hline M1 $(n=17)$ & $406.321 \pm 125.660$ & $333.806 \pm 126.705$ & $426.979 \pm 110.778$ & $379.487 \pm 121.888$ & 1.104 & 0.359 \\
M2 $(n=42)$ & $409.166 \pm 113.272$ & $369.613 \pm 103.376$ & $379.477 \pm 95.950$ & $387.974 \pm 105.969$ & 0.638 & 0.534 \\
M3 $(n=28)$ & $421.006 \pm 89.904$ & $374.321 \pm 104.572$ & $362.879 \pm 53.050$ & $388.542 \pm 91.089$ & 1.021 & 0.375 \\
Total & $412.710 \pm 103.840$ & $363.562 \pm 106.982$ & $389.778 \pm 89.029$ & $386.498 \pm 103.608$ & 1.976 & 0.145 \\
F & 0.045 & 0.386 & 0.820 & 0.048 & 0.954 \\
P & 0.956 & 0.683 & 0.459 & & \\
\hline
\end{tabular}

$\mathrm{n}$ : the number of thrombi.
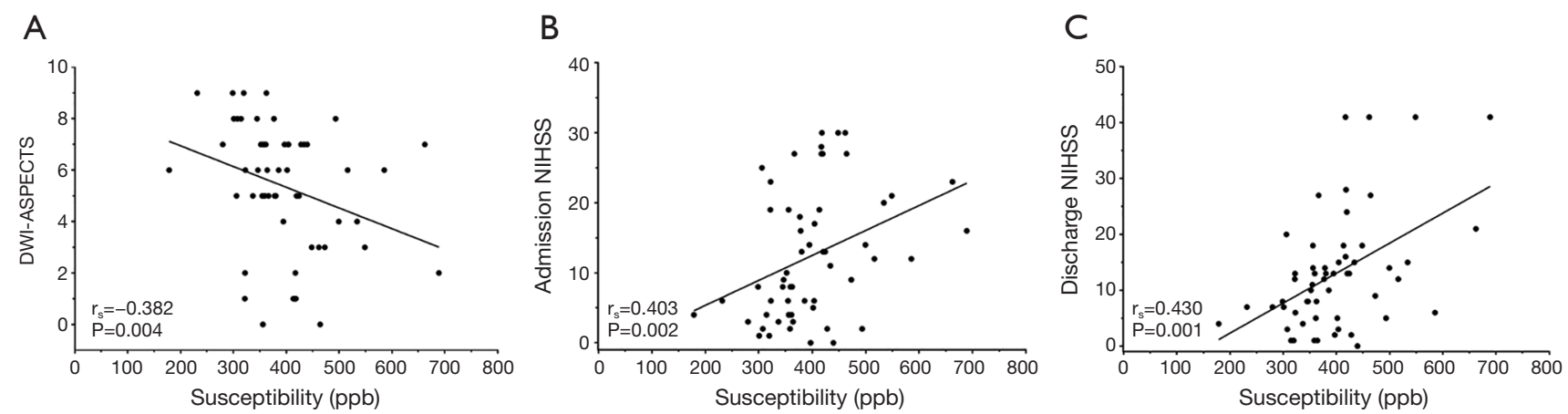

Figure 4 The point-and-figure charts of relationship between thrombus susceptibility and diffusion-weighted imaging-Alberta stroke program early CT score (DWI-ASPECTS), admission National Institutes of Health Stroke Scale (NIHSS), discharge NIHSS. (A) The susceptibility was weakly negatively correlated with DWI-ASPECTS $\left(\mathrm{r}_{\mathrm{s}}=-0.382, \mathrm{P}=0.004\right)$. (B) The susceptibility was weakly yet positively correlated with NIHSS at admission $\left(r_{s}=0.403, P=0.002\right)$ and $(C)$ weakly yet positively correlated with NIHSS at discharge $\left(r_{s}=0.430\right.$, $\mathrm{P}=0.001)$.

thrombosis, which could be used as an independent factor in predicting the cerebral function post-infarction $(36,39)$.

This study found a weak correlation between thrombus length of MCA and admission NIHSS, but no correlation with discharge NIHSS. The longer the thrombus, the more severe the clinical status. The admission NIHSS was related to the area and location of cerebral infarction. Yet, the discharge NIHSS was not only affected by the admission NIHSS score but was also affected by patient treatment. As a result, thrombus length might not be considered an independent predictor of short-term clinical outcome of cerebral infarction.

We found that CBS associated with the portion of MCA thrombus was not only weakly yet positively associated with DWI-ASPECTS, but also weakly yet negatively correlated with admission and discharge NIHSS. It was verified that the thrombus load was not only related to the thrombus length but also inseparable from the location of the thrombus $(5,33,34)$. When thrombus occurred only in MCA branch, CBS was higher than those occurring only in the MCA trunk. Therefore, thrombus occurring in the MCA branch might cause a smaller range of cerebral infarction and less brain damage than thrombus occurring in the trunk. The less the damage after infarction, the better the short-term clinical prognosis.

There were three shortcomings in the current research. First, there was no follow-up data to show the natural evolution of thrombus on SWI. Second, there was no available pathology from thrombectomy to support the consistent susceptibility with a similar component. Third, there was no 3-month follow-up after to evaluate the longterm prognosis of the patients. Therefore, more data should be collected to show the natural evolution and changes of susceptibility during this processing, and the relationship 


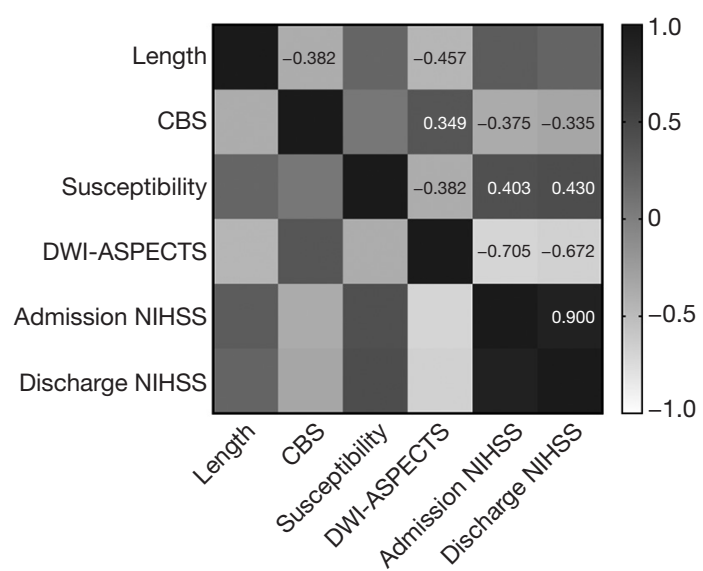

Figure 5 The relationship and correlation coefficient of length, clot burden score (CBS), susceptibility of thrombus, diffusionweighted imaging-Alberta stroke program early CT score (DWIASPECTS), admission National Institutes of Health Stroke Scale (NIHSS), and discharge NIHSS of stroke patients. The susceptibility and length were weakly yet negatively correlated with DWI-ASPECTS $\left(\mathrm{r}_{\mathrm{s}}=-0.382,-0.457 ; \mathrm{P}=0.004,0.000\right)$. The susceptibility was weakly yet positively correlated with admission NIHSS and discharge NIHSS $\left(r_{s}=0.403,0.430 ; \mathrm{P}=0.002,0.001\right)$. CBS was weakly yet positively correlated with DWI-ASPECTS $\left(r_{s}=0.349, P=0.008\right)$ and weakly yet negatively correlated with admission and discharge NIHSS $\left(r_{s}=-0.375,-0.335 ; P=0.004\right.$, 0.012). The CBS was weakly yet negatively correlated with apparent thrombus length of middle cerebral artery (MCA) $\left(r_{s}=-0.382, P=0.004\right)$.

between susceptibility and long-term prognosis.

In conclusion, susceptibility remained consistent regardless of the different segments, portions, or onset time, which indicates a similar component of whole thrombus and formation stage. Susceptibility can be an important parameter related to cerebral infarction along with CBS, and they may, to some extent, help predict clinical severity and short-term clinical prognosis

\section{Acknowledgments}

Funding: This work was supported by the Natural Scientific Foundation of China (grant number 81871342 to S Xia; grant number 81901728 to $\mathrm{C}$ Chai) and the Chronic Disease Prevention from Tianjin Scientific Funding (grant number 16ZXMJSY00210 to S Xia).

\section{Footnote}

Conflicts of Interest: The authors have no conflicts of interest to declare.

Ethical Statement: The current study was approved by the local Institutional Review Board (IRB) of Tianjin First Central Hospital. Written consent signed by the patients or their relatives were stored in the hospital database to be reviewed.

\section{References}

1. Furlan A, Higashida R, Wechsler L, Gent M. Rowley H, Kase C, Pessin M, Ahuja A, Callahan F, Clark WM, Silver $\mathrm{F}$, Rivera F. Intra-arterial prourokinase for acute ischemic stroke. The PROACT II study: a randomized controlled trial. Prolyse in Acute Cerebral Thromboembolism. JAMA 1999;282:2003-11.

2. del Zoppo GJ, Poeck K, Pessin MS, Wolpert SM, Furlan AJ, Ferbert A, Alberts MJ, Zivin JA, Wechsler L, Busse O. Recombinant tissue plasminogen activator in acute thrombotic and embolic stroke. Ann Neurol 1992;32:78-86.

3. Jörgensen L, Torvik A. Ischaemic cerebrovascular diseases in an autopsy series. I. Prevalence, location and predisposing factors inverified thrombo-embolic occlusions, and their significance in the pathogenesis of cerebral infarction. J Neurol Sci 1966;3:490-509.

4. Cho KH, Kim JS, Kwon SU, Cho AH, Kang DW. Significance of Susceptibility Vessel Sign on T2* Weighted Gradient Echo Imaging for Identification of Stroke Subtypes. Stroke 2005;36:2379-83.

5. Legrand L, Naggara O, Turc G, Mellerio C, Roca P, Calvet D, Labeyrie MA, Baron JC, Mas JL, Meder JF, Touzé E, Oppenheim C. Clot burden score on admission T2*-MRI predicts recanalization in acute stroke. Stroke 2013;44:1878-84.

6. Riedel CH, Zimmermann P, Jensen-Kondering U, Stingele R, Deuschl G, Jansen O. The importance of size: successful recanalization by intravenous thrombolysis in acute anterior stroke depends on thrombus length. Stroke 2011;42:1775-7.

7. Krings T, Noelchen D, Mull M, Willmes K, Meister IG, Reinacher P, Toepper R, Thron AK. The hyperdense posterior cerebral artery sign: a computed tomography marker of acute ischemia in the posterior cerebral artery 
territory. Stroke 2006;37:399-403.

8. Ambrosius W, Gupta V, Kazmierski R, Hellmann A, Qian G, Nowinski WL. The hyperdense posterior cerebral artery sign in CT is related to larger ischemic lesion volume. Pol J Radiol 2011;76:13-7.

9. Leary MC, Kidwell CS, Villablanca JP, Starkman S, Jahan R, Duckwiler GR, Gobin YP, Sykes S, Gough KJ, Ferguson K, Llanes JN, Masamed R, Tremwel M, Ovbiagele B, Vespa PM, Vinuela F, Saver JL. Validation of computed tomographic middle cerebral artery "dot" sign: an angiographic correlation study. Stroke 2003;34:2636-40.

10. Liebeskind DS, Sanossian N, Yong WH, Starkman S, Tsang MP, Moya AL, Zheng DD, Abolian AM, Kim D, Ali LK, Shah SH, Towfighi A, Ovbiagele B, Kidwell CS, Tateshima S, Jahan R, Duckwiler GR, Viñuela F, Salamon N, Villablanca JP, Vinters HV, Marder VJ, Saver JL. CT and MRI early vessel signs reflect clot composition in acute stroke. Stroke 2011;42:1237-43.

11. Radbruch A, Mucke J, Schweser F, Deistung A, Ringleb PA, Ziener CH, Roethke M, Schlemmer HP Heiland S, Reichenbach JR, Bendszus M, Rohde S. Comparison of Susceptibility Weighted Imaging and TOF-Angiography for the Detection of Thrombi in Acute Stroke. PLoS One 2013;8:e63459.

12. Park MG, Yoon CH, Baik SK. Susceptibility Vessel Sign for Intra-arterial Thrombus in Acute Posterior Cerebral Artery Infarction. J Stroke Cerebrovasc Dis 2015;24:1229-34.

13. Allibert R, Billon Grand C, Vuillier F, Cattin F, Muzard E, Biondi A, Moulin T, Medeiros E. Advantages of susceptibility-weighted magnetic resonance sequences in the visualization of intravascular thrombi in acute ischemic stroke. Int J Stroke 2014;9:980-4.

14. Kang DW, Jeong HG, Kim DY, Yang W, Lee SH. Prediction of Stroke Subtype and Recanalization Using Susceptibility Vessel Sign on Susceptibility-Weighted Magnetic Resonance Imaging. Stroke 2017;48:1554-59.

15. Flacke S, Urbach H, Keller E, Träber F, Hartmann A, Textor J, Gieseke J, Block W, Folkers PJ, Schild H H. Middle cerebral artery (MCA) susceptibility sign at susceptibility-based perfusion MR imaging: clinical importance and comparison with hyperdense MCA sign at CT. Radiology 2000;215:476-82.

16. Aoki J, Kimura K, Shibazaki K, Saji N, Uemura J, Sakamoto Y, Nagai K. The susceptibility vessel sign at the proximal M1: a strong predictor for poor outcome after intravenous thrombolysis. J Neurol Sci 2015;348:195-200.
17. Aoki J, Kimura K, Shibazaki K, Sakamoto Y, Saji N, Uemura J. Location of the Susceptibility Vessel Sign on T2*-Weighted MRI and Early Recanalization within 1 Hour after Tissue Plasminogen Activator Administration. Cerebrovasc Dis Extra 2013;3:111-20.

18. Park MG, Oh SJ, Baik SK, Jung DS, Park KP. Susceptibility-Weighted Imaging for Detection of Thrombus in Acute Cardioembolic Stroke. J Stroke 2016;18:73-9.

19. Gratz PP, Schroth G, Gralla J, Mattle HP, Fischer U, Jung S, Mordasini P, Hsieh K, Verma RK, Weisstanner C, El-Koussy M. Whole-Brain Susceptibility-Weighted Thrombus Imaging in Stroke: Fragmented Thrombi Predict Worse Outcome. AJNR Am J Neuroradiol 2015;36:1277-82.

20. Payabvash S, Benson JC, Taleb S, Rykken JB, Hoffman B, McKinney AM, Oswood MC. Susceptible vessel sign: identification of arterial occlusion and clinical implications in acute ischaemic stroke. Clin Radiol 2017;72:116-122.

21. Shinohara Y, Kinoshita T, Kinoshita F. Changes in susceptibility signson serial T2*-weighted single-shot echo-planar gradient-echo images in acute embolic infarction: comparison with recanalization status on 3D time-of-flight magnetic resonance angiography. Neuroradiology 2012;54:427-34.

22. Langkammer C, Schweser F, Krebs N, Deistung A, Goessler W, Scheurer E, Sommer K, Reishofer G, Yen K, Fazekas F, Ropele S, Reichenbach JR. Quantitative susceptibility mapping (QSM) as a means to measure brain iron? Apost mortem validation study. Neuroimage 2012;62:1593-9.

23. Zivadinov R, Weinstock-Guttman B, Pirko I. Iron deposition and inflammation in multiplesclerosis. Which one comes first? BMC Neurosci 2011;12:60.

24. Qiu D, Chan GC, Chu J, Chan Q, Ha SY, Moseley ME, Khong PL. MR quantitative susceptibility imaging for the evaluation of iron loading in the brains of patients with $\beta$-thalassemia major. AJNR Am J Neuroradiol 2014;35:1085-90.

25. Langkammer C, Liu T, Khalil M, Enzinger C, Jehna M, Fuchs S, Fazekas F, Wang Y, Ropele S. Quantitative susceptibility mapping in multiple sclerosis. Radiology 2013;267:551-9.

26. Hermier M, Nighoghossian N, Derex L, Berthezène Y, Blanc-Lasserre K, Trouillas P, Froment JC.MRI of acute post-ischemic cerebral hemorrhage in stroke patients: diagnosis with T2*-weighted gradient-echo sequences. Neuroradiology 2001;43:809-15. 
27. Liu T, Surapaneni K, Lou M, Cheng L, Spincemaille P, Wang Y. Cerebral microbleeds: burden assessment by using quantitative susceptibility mapping. Radiology 2012;262:269-78.

28. Xia S, Utriainen D, Tang J, Kou Z, Zheng G, Wang $\mathrm{X}$, Shen W, Haacke EM. Lu G. Decreased oxygen saturationin asymmetrically prominent cortical veins in patients with cerebral ischemic stroke. Magn Reson Imaging 2014;32:1272-6.

29. Li M, Hu J, Miao Y, Shen H, Tao D, Yang Z, Li Q, Xuan SY, Raza W, Alzubaidi S, Haacke EM. In vivo measurement of oxygenation changes after stroke using susceptibility weighted imaging filtered phase data. PLoS One 2013;8:e63013.

30. Zhang J, Liu T, Gupta A, Spincemaille P, Nguyen TD, Wang Y. Quantitative mapping of cerebral metabolic rate of oxygen (CMRO2) using quantitative susceptibility mapping (QSM). Magn Reson Med 2015;74:945-52.

31. Smith SM. Fast robust automated brain extraction. Hum Brain Mapp 2002;17:143-55.

32. Haacke EM, Tang J, Neelavalli J, Cheng YC. Susceptibility mapping as a means to visualize veins and quantify oxygen saturation. J Magn Reson Imaging 2010;32:663-76.

33. Tan IY, Demchuk AM, Hopyan J, Zhang L, Gladstone D, Wong K, Martin M, Symons SP, Fox AJ, Aviv RI. CT angiography clot burden score and collateral score: correlation with clinical and radiologic outcomes in acute middle cerebral artery infarct. AJNR Am J Neuroradiol 2009;30:525-31

34. Puetz V, Dzialowski I, Hill MD, Subramaniam S, Sylaja

Cite this article as: Zheng MZ, Yang QY, Lu XD, Hu SL, Chai C, Shen W, Chang BG, Wang ZY, Xia S. Middle cerebral artery thrombus susceptibility-weighted imaging mapping predicts prognosis. Quant Imaging Med Surg 2019;9(9):15561565. doi: 10.21037/qims.2019.08.17
PN, Krol A, O'Reilly C, Hudon ME, Hu WY, Coutts SB, Barber PA, Watson T, Roy J, Demchuk AM; Calgary CTA Study Group. Intracranial thrombus extent predicts clinical outcome, final infarct size and hemorrhagic transformation in ischemic stroke: the clot burden score. Int J Stroke 2008;3:230-6.

35. Hill MD, Rowley HA, Adler F, Eliasziw M, Furlan A, Higashida RT, Wechsler LR, Roberts HC, Dillon WP, Fischbein NJ, Firszt CM, Schulz GA, Buchan AM; PROACT-II Investigators. Selection of acute ischemic stroke patients for intra-arterial thrombolysis with prourokinase by using ASPECTS. Stroke 2003;34:1925-31.

36. Aoki J, Kimura K, Shibazaki K, Sakamoto Y. DWIASPECTS as a predictor of dramatic recovery after intravenous recombinant tissue plasminogen activator administration in patients with middle cerebral artery occlusion. Stroke 2013;44:534-7.

37. Marder VJ, Chute DJ, Starkman S, Abolian AM, Kidwell C, Liebeskind D, Ovbiagele B, Vinuela F, Duckwiler G, Jahan R, Vespa PM, Selco S, Rajajee V, Kim D, Sanossian $\mathrm{N}$, Saver JL. Analysis of thrombi retrieved from cerebral arteries of patients with acute ischemic stroke. Stroke 2006;37:2086-93.

38. Hayman LA, Taber KH, Ford JJ, Saleem A, Gurgun M, Mohamed S, Bryan RN. Effect of clot formation and retraction on spin-echo MR images of blood: an in vitro study. AJNR Am J Neuroradiol 1989;10:1155-58.

39. Tei H, Uchiyama S, Usui T, Ohara K. Diffusion-weighted ASPECTS as an independent marker for predicting functional outcome. J Neurol 2011;258:559-65. 


\section{Supplementary}

Table S1 Consistency test of measurement indicators

\begin{tabular}{lcc}
\hline Indicators & ICC $(95 \%$ CI $)$ & $P$ \\
\hline Length of thrombus & $0.959(0.919-0.978)$ & $<0.001$ \\
Reader A & $0.958(0.927-0.976)$ & $<0.001$ \\
Reader B & $0.957(0.921-0.976)$ & $<0.001$ \\
Reader A and Reader B & & \\
Susceptibility & $0.957(0.925-0.975)$ & $<0.001$ \\
Reader A & $0.958(0.927-0.976)$ & $<0.001$ \\
Reader B & $0.945(0.657-0.981)$ & $<0.001$ \\
\hline
\end{tabular}

Reader A, the 10-year experienced neuroradiologist; Reader B, the 5-year experienced neuroradiologist.

Table S2 K-S test for normal distribution of variables

\begin{tabular}{lcccccccc}
\hline Variables & Age & Onset time & Length & CBS & Susceptibility & DWI-ASPECTS & Admission NIHSS & Discharge NIHSS \\
\hline Variable type & CNV & CNV & CNV & CNV & CNV & CNV & CNV & CNV \\
P & 0.200 & 0.000 & 0.001 & 0.000 & 0.200 & 0.002 & 0.018 & 0.000 \\
Distribution & Normal & Non & Non & Non & Normal & Non & Non & Non \\
\hline
\end{tabular}

CBS, clot burden score; DWI-ASPECTS, diffusion-weighted imaging-Alberta stroke program early CT score; NIHSS, National Institutes of Health Stroke Scale; CNV, continuous numerical variables; Non, non-normal distribution.

Table S3 The correlation coefficient between variables

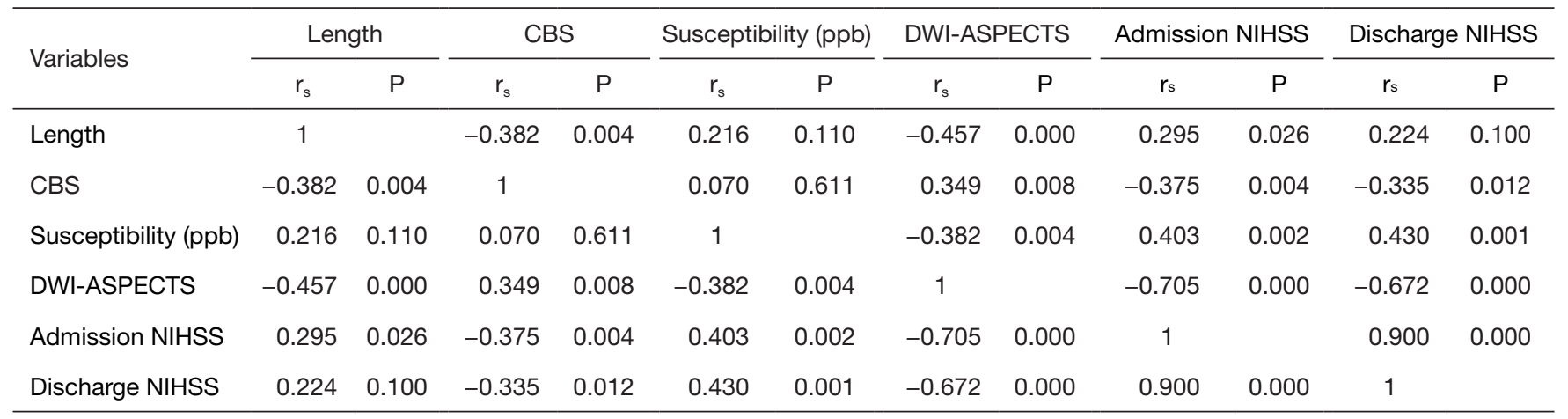

CBS, clot burden score; DWI-ASPECTS, diffusion-weighted imaging-Alberta stroke program early CT score; NIHSS, National Institutes of Health Stroke Scale. 
A

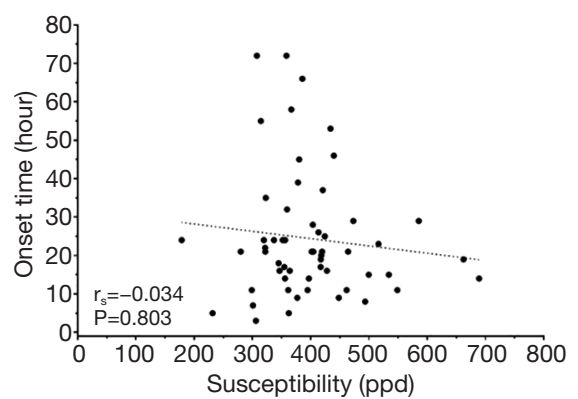

B

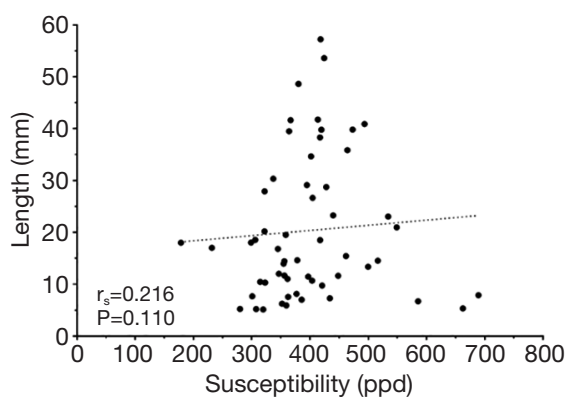

C

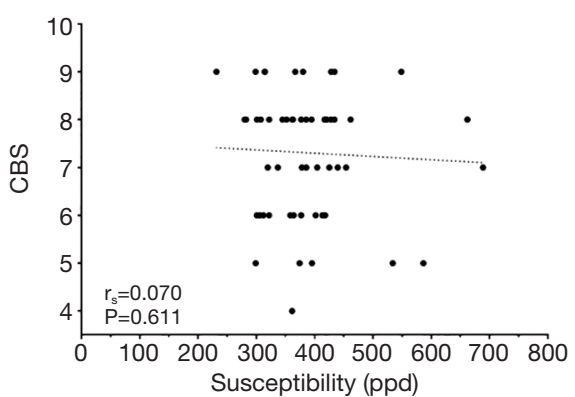

Figure S1 The point-and-figure charts between thrombus susceptibility and the onset time, thrombus length, clot burden score (CBS). No correlation was found between susceptibility and the onset time (A), thrombus length (B), and CBS (C) $(\mathrm{P}=0.803,0.110,0.611)$.

A

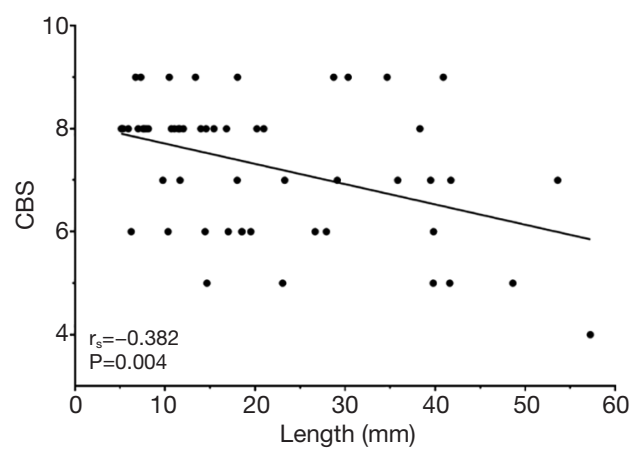

C

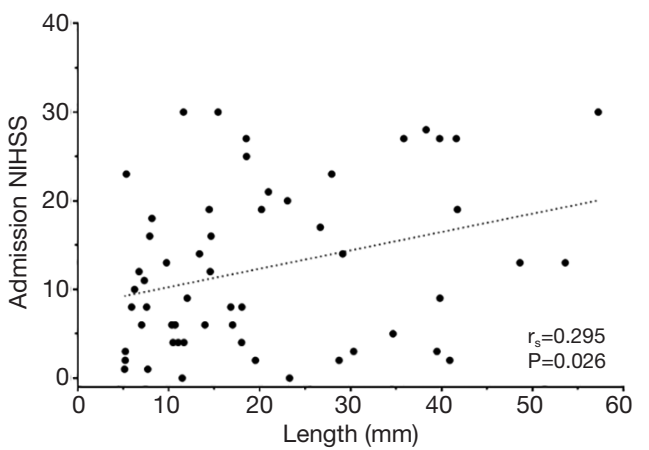

B

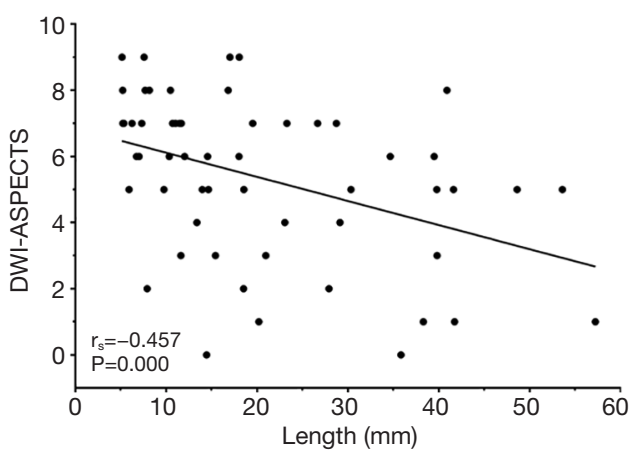

$\mathrm{D}$

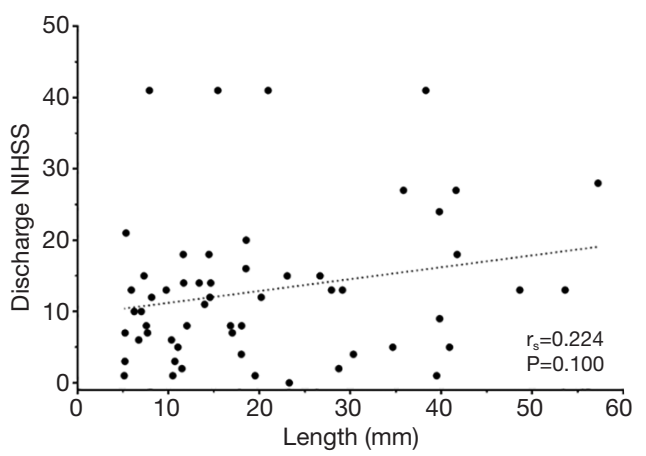

Figure S2 The point-and-figure charts of relationship between thrombus length and other variables. (A) The clot burden score (CBS) was weakly yet negatively correlated with thrombus length of middle cerebral artery (MCA) $\left(r_{s}=-0.382, P=0.004\right)$. (B) The length of MCA thrombus was negatively correlated with diffusion-weighted imaging-Alberta stroke program early CT score (DWI-ASPECTS) ( $\mathrm{r}_{\mathrm{s}}=-0.457$, $\mathrm{P}=0.000)$. (C,D) There was no statistically significant correlation between the length of MCA thrombus and admission and discharge National Institutes of Health Stroke Scale (NIHSS) $\left(r_{s}=0.295,0.224 ; \mathrm{P}=0.026,0.100\right)$..

A

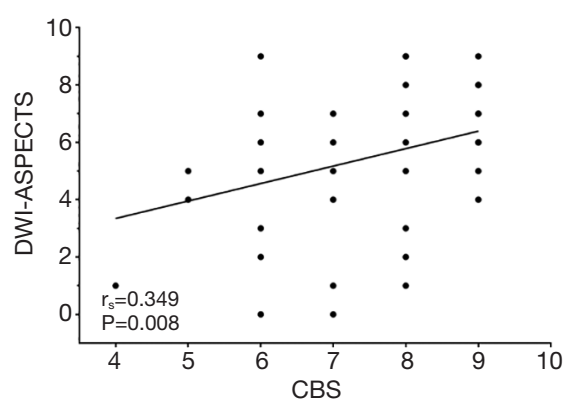

B

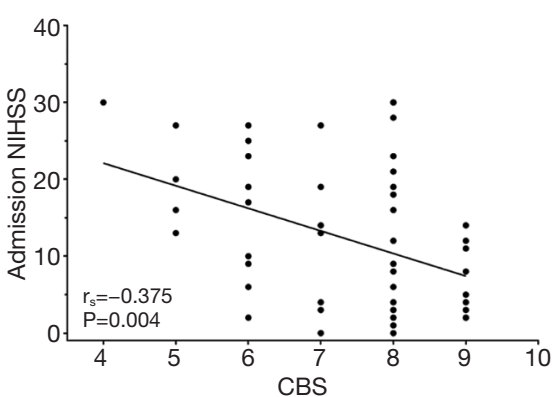

C

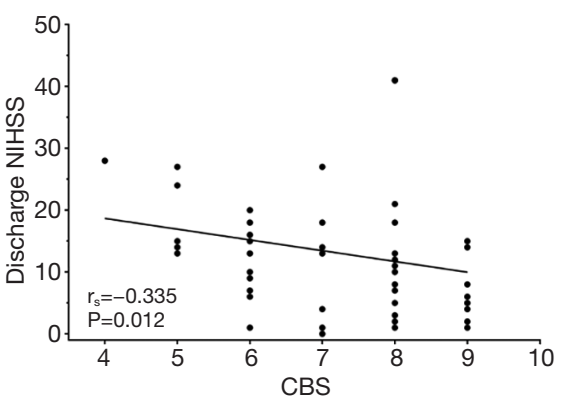

Figure S3 The point-and-figure charts of relationship between clot burden score (CBS) and diffusion-weighted imaging-Alberta stroke program early CT score (DWI-ASPECTS), admission National Institutes of Health Stroke Scale (NIHSS), discharge NIHSS. (A) CBS was weakly yet positively correlated with DWI-ASPECTS $\left(\mathrm{r}_{\mathrm{s}}=0.349, \mathrm{P}=0.008\right)$; (B,C) CBS was weakly yet negatively correlated with the admission and discharge NIHSS $\left(\mathrm{r}_{\mathrm{s}}=-0.375,-0.338 ; \mathrm{P}=0.004,0.012\right)$. 